\title{
Rational tangle surgery and Xer recombination on catenanes
}

\author{
ISABEL K DARCY \\ KAI ISHIHARA \\ RAM K MEDIKONDURI \\ KOYA SHIMOKAWA
}

\begin{abstract}
The protein recombinase can change the knot type of circular DNA. The action of a recombinase converting one knot into another knot is normally mathematically modeled by band surgery. Band surgeries on a $2-$ bridge knot $N\left(\frac{4 m n-1}{2 m}\right)$ yielding a $(2,2 k)$-torus link are characterized. We apply this and other rational tangle surgery results to analyze Xer recombination on DNA catenanes using the tangle model for protein-bound DNA.
\end{abstract}

$57 \mathrm{M} 25,92 \mathrm{C} 40$

\section{Introduction}

A tangle consists of arcs properly embedded in a three dimensional ball. A proteinDNA complex can be regarded as a tangle in which the protein complex is considered as a three dimensional ball and the DNA within the complex as arcs (see Ernst and Sumners [12] and Sumners, Ernst, Spengler and Cozzarelli [30]). In general it is very hard to identify the arrangement of DNA within a protein-DNA complex. Electron micrographs, AFM (Atomic Force Microscopy) images, and crystalline structures do not give clear enough data to address this problem for large molecules. Thus the tangle model is frequently used in analyzing the topology of protein-bound DNA (see Buck and Verjovsky Marcotte [2], Darcy and Sumners [9], Ernst and Sumners [12; 13] and Vazquez and Sumners [32]).

The proteins we are interested in are ones which are involved in recombination. Sitespecific recombination is a process in which specific target sequences on each of two DNA segments are exchanged. These specific sites are called recombination sites. Proteins that carry out these recombination reactions are called recombinases. Site-specific recombination reactions are involved in a variety of biological processes including transposition of DNA, integration into host chromosomes, and gene regulation.

During recombination, the topology of circular DNA can change forming knots and links. The local action of a recombinase has been modeled by the mathematical operation 
of a band surgery or a rational tangle surgery. In this paper we will characterize such surgeries on a 2 -bridge knot $N\left(\frac{4 m n-1}{2 m}\right)$ yielding a $(2,2 k)$-torus link. The class of knots $N\left(\frac{4 m n-1}{2 m}\right)$ includes the family of twist knots which frequently appear in biological reactions. It also includes knots which are believed to be the products of Xer recombination when Xer acts on $(2,2 k)$-torus links.

In Section 2, we give mathematical preliminaries. In Section 3, we state the main result which we apply to Xer recombination in Section 4. We prove our main result in Section 5. In Section 6 we consider non-band rational tangle surgery cases related to Xer recombination. In Section 7 we summarize our results and briefly discuss the software TopoIce-R [8] within Knotplot [26] which implements the results of Theorem 3.1.

\section{Tangles and 2-bridge knots and links}

Let $T, T_{1}$, and $T_{2}$ be 2 -string tangles. The knot (or link) obtained by connecting the top two endpoints of $T$ and the bottom two endpoints by simple curves, as shown in Figure 1, is called the numerator closure and is denoted by $N(T)$. From two tangles $T_{1}$ and $T_{2}$ a new tangle, called the sum of $T_{1}$ and $T_{2}$ and denoted by $T_{1}+T_{2}$, can be obtained by connecting two endpoints of $T_{1}$ to two endpoints of $T_{2}$ as shown in Figure 1. For more on tangles see Conway [4], Goldman and Kauffman [14], Murasugi [25] and Sumners, Ernst, Spengler and Cozzarelli [30].
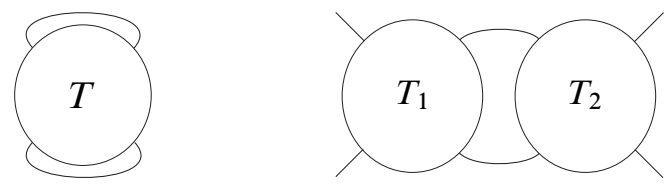

Figure 1. The numerator $N(T)$ (left) and the tangle sum $T_{1}+T_{2}$ (right)

Let $c_{1}, \ldots, c_{n}$ be a sequence of integers. The circle product of a tangle $T$ and $\left(c_{1}, \ldots, c_{n}\right)$ is defined as shown in Figure 2 and is denoted by $T \circ\left(c_{1}, \ldots, c_{n}\right)$ (see Darcy [7]). A rational tangle is the circle product of a zero crossing tangle and $\left(c_{1}, \ldots, c_{n}\right)$. The zero crossing tangle contains two vertical strings if $n$ is even or two horizontal strings if $n$ is odd. Rational tangles are classified up to ambient isotopy fixing the boundary by their continued fraction

$$
c_{n}+\frac{1}{c_{n-1}+\frac{1}{\cdots+\frac{1}{c_{1}}}}
$$

(see Conway [4]). 

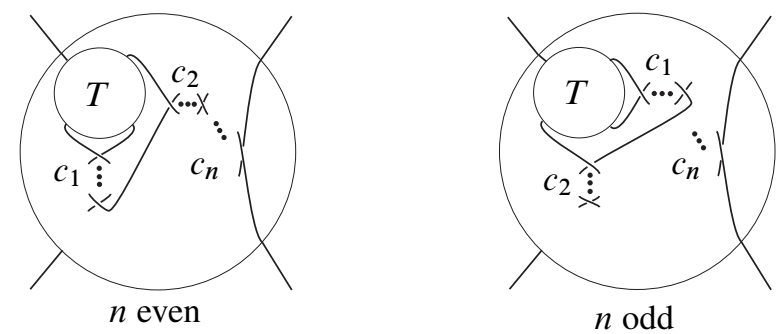

Figure 2. Circle products $T \circ\left(c_{1}, \ldots, c_{n}\right)$ of $T$ and $\left(c_{1}, \ldots, c_{n}\right)$ : The vertical twists are left-handed if $c_{i}>0$ and right-handed if $c_{i}<0$ while the horizontal twists are right-handed if $c_{i}>0$ and left-handed if $c_{i}<0$.

The knot/link, $N\left(\frac{a}{b}\right)$ can be formed from the rational tangle $\frac{a}{b}$ via numerator closure. $N\left(\frac{a}{b}\right)$ is a 2-bridge knot/link except when $|a|=1$ in which case it is the unknot. $N\left(\frac{a}{b}\right)$ is a link if and only if $a$ is even in which case it is a 2-component link. The 2-component link $N(2 k)$ is called a $(2,2 k)$-torus link. Given a projection of a link, the linking number of a 2-component link can be determined by calculating the sum over all crossings involving both components of the signed crossing number.

Let $P$ and $R$ be rational tangles. The operation which transforms a knot (or link) by replacing $P$ with $R$ is called a rational tangle surgery, and we will refer to this as a $(P, R)$ move. See Figure 3. A $(P, R)$ move is said to be equivalent to a $\left(P^{\prime}, R^{\prime}\right)$ move if and only if for every pair of knots, $K_{1}$ and $K_{2}$, there exists $U$ satisfying the system of tangle equations $N(U+P)=K_{1}, N(U+R)=K_{2}$ if and only if there exists $U^{\prime}$ satisfying the system of tangle equations $N\left(U^{\prime}+P^{\prime}\right)=K_{1}, N\left(U^{\prime}+R^{\prime}\right)=K_{2}$. The following two theorems classify equivalent $(P, R)$ moves when $P$ and $R$ are rational tangles.

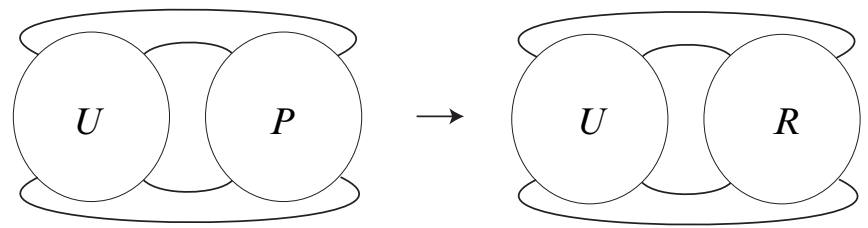

Figure 3. A rational tangle surgery: $P$ and $R$ are rational 2-string tangles. $U$ is a $2-$ string tangle.

Theorem 2.1 (Darcy [7]) A $\left(0, \frac{t}{w}\right)$ move is equivalent to a $\left(0, \frac{c}{d}\right)$ move if and only if $\frac{c}{d}=\frac{t}{w-h t}$ for some $h$. Moreover, $N\left(U+\frac{0}{1}\right)=K_{1}$ and $N\left(U+\frac{t}{w}\right)=K_{2}$ if and only if $N\left([U \circ(h, 0)]+\frac{0}{1}\right)=K_{1}$ and $N\left([U \circ(h, 0)]+\frac{t}{w-h t}\right)=K_{2}$. 
Theorem 2.2 (Darcy [7]) An $\left(\frac{f_{1}}{g_{1}}, \frac{f_{2}}{g_{2}}\right)$ move is equivalent to a $\left(0, \frac{t}{w}\right)$ move if and only if there exists $e_{1}$ and $i_{1}$ such that $g_{1} e_{1}-f_{1} i_{1}=1$ and $\frac{t}{w}=\frac{g_{1} f_{2}-g_{2} f_{1}}{e_{1} g_{2}-i_{1} f_{2}}$ (or equivalently, $\frac{f_{2}}{g_{2}}=\frac{t e_{1}+w f_{1}}{t i_{1}+w g_{1}}$ ).

As discussed in [7], any solution for $U$ to $N(U+P)=K_{1}, N(U+R)=K_{2}$ can be translated into a solution for $U^{\prime}$ to $N\left(U^{\prime}+0\right)=K_{1}, N\left(U^{\prime}+R^{\prime}\right)=K_{2}$ and vice versa. Hence we will often focus on the $P=0$ case.

Let $L$ be a link in $S^{3}$. Let $b: I \times I \rightarrow S^{3}$ be a band satisfying $b^{-1}(L)=I \times \partial I$, where $I=[0,1]$ is an interval. Let $L_{b}$ denote a link obtained by replacing $b(I \times \partial I)$ in $L$ with $b(\partial I \times I)$. For simplicity we denote $b(I \times I)$ by $b$. We say $L_{b}$ is obtained from $L$ by a band surgery along $b$. Note that band surgery is equivalent to rational tangle surgery where $P=0$ and $R=\frac{1}{w}$ for some integer $w$. If $L$ and $L_{b}$ have orientations which agree with each other except for the band $b$, the corresponding band surgery is said to be coherent. A $\left(0, \frac{1}{w}\right)$ move can be considered as a band surgery. See Figure 4 . Note that by choosing the orientations of $L$ and $L_{b}$, any band surgery can be a coherent one with respect to those orientations.

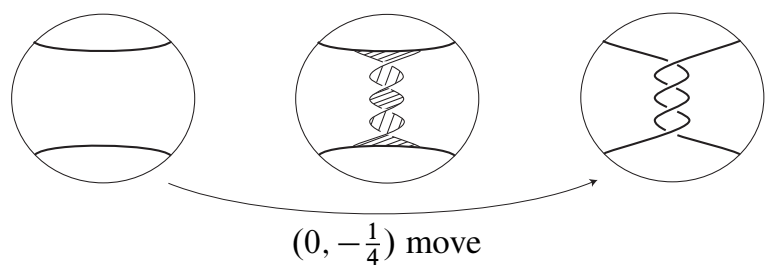

Figure 4. A band surgery and a $\left(0, \frac{1}{w}\right)$ move

\section{Statement of the main theorem: characterization of band surgery}

In this section we state a result characterizing band surgeries on a 2-bridge knot $N\left(\frac{4 m n-1}{2 m}\right)$ yielding a $(2,2 k)$-torus link $N(2 k)$.

\section{Theorem 3.1 Suppose}

(*) $\quad N(U+0)=N\left(\frac{4 m n-1}{2 m}\right)$

and

$N\left(U+\frac{1}{w}\right)=(2,2 k)$-torus link

and the rational tangle surgery corresponds to a coherent band surgery. If the $(2,2 k)-$ torus link has linking number $k$ where $|k|>2$, then $(*)$ has no solution. If the $(2,2 k)$-torus link has linking number $-k$, then one of the following holds (see Figure 5 below): 
(1) $k=m$ and $U=\left(\frac{4 m n-1}{-w(4 m n-1)+2 m}\right)$.

(2) $k=n$ and $U=\left(\frac{4 m n-1}{-w(4 m n-1)+2 n}\right)$.

(3) $k=m+n+1$ and $U=\left(\frac{-1}{2 m+1}+\frac{-1}{2 n+1}\right) \circ(1,-(w+1), 0)$. or $U=\left(\frac{-1}{2 n+1}+\frac{-1}{2 m+1}\right) \circ(1,-(w+1), 0)$.

(4) $k=m+n-1$ and $U=\left(\frac{-1}{2 m-1}+\frac{-1}{2 n-1}\right) \circ(-1,-(w-1), 0)$.

$$
\text { or } U=\left(\frac{-1}{2 n-1}+\frac{-1}{2 m-1}\right) \circ(-1,-(w-1), 0) \text {. }
$$

Note if the $(2, \pm 4)$-torus link has linking number \pm 2 where the \pm signs agree, then we do not know if $(*)$ has a solution corresponding to a coherent band surgery. See Section 4.4 for a case where the $(2,4)$-torus link has linking number 2 and the product is the trefoil knot. However, if the $(2, \pm 4)$-torus link has linking number $\mp 2$, then we can use Theorem 3.1 to determine all solutions (if any) to $(*)$. The Hopf link = the $(2,2)$-torus link $=$ the $(2,-2)$-torus link. Thus Theorem 3.1 applies to the Hopf link no matter how it is oriented. When $k=0, N(2 k)=N(0)=$ unlink of two components. Hence Theorem 3.1 also applies to the two component unlink.

The case where $m n=0$ (that is, $N\left(\frac{4 m n-1}{2 m}\right)$ is an unknot) was proved by Hirasawa and Shimokawa [17]. In Section 5, we prove Theorem 3.1 for the case where $m, n \neq 0$.

\section{Results on Xer recombination on DNA catenanes}

Recall that recombinases are proteins which bind to and break two segments of DNA, exchange the ends of the broken DNA before resealing the breaks (Figure 6). Since recombinases normally bind to asymmetric recombination sites, the DNA sequence of the recombination sites can be used to orient these sites as shown in Figure 6. When the two sites are on the same component, their orientations on the circular DNA molecule can either agree (Figure 6A) or disagree (Figure 6B). In the former case, we say that the two sites are directly repeated, while in the later case, we say that the two sites are inversely repeated. Observe that recombination on directly repeated sites normally results in a change in the number of components while for inversely repeated sites the number of components is normally preserved.

When a site-specific recombinase binds to two segments of circular DNA, the DNA can be partitioned in different ways. Sometimes one partitions the DNA into two tangles where one tangle represents the DNA bound by the protein while the other tangle represents the free DNA not bound by protein. In other cases, we are interested in the local action of the DNA. In this case, one tangle represents the two very short segments of DNA upon which the recombinase acts, breaking, exchanging and rejoining the DNA segments. This local recombination reaction is modeled by replacing the tangle 
(1)

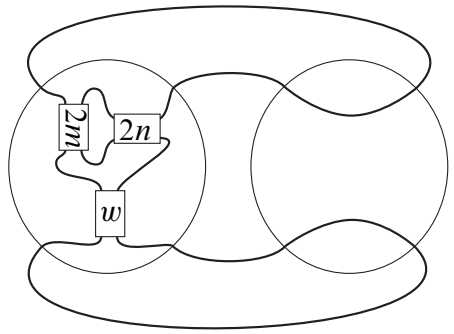

(2)

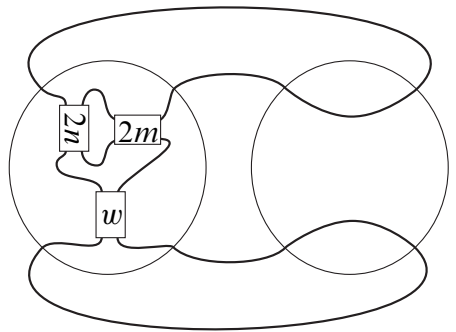

(3)

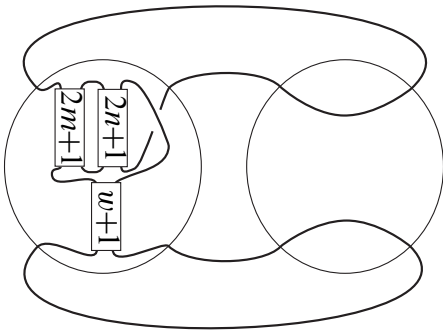

(4)

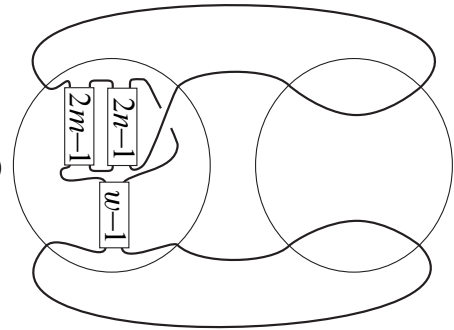

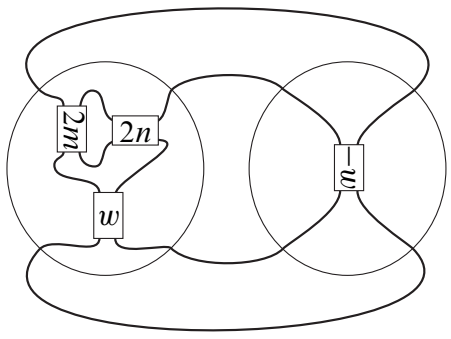
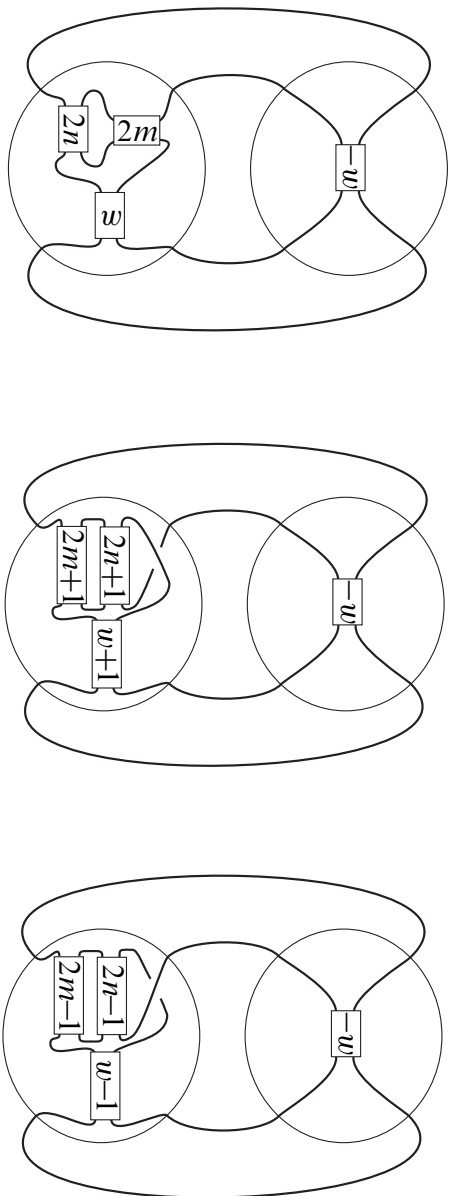

Figure 5. Rational tangle surgeries from $N\left(\frac{4 m n-1}{2 m}\right)$ to $N(2 k)$ : A positive (negative) number inside a box corresponds to right (left) handed twists. For example, $N\left(\frac{4 m n-1}{2 m}\right)=(-2 m, 2 n,-w, 0)$ per left side of (1). 


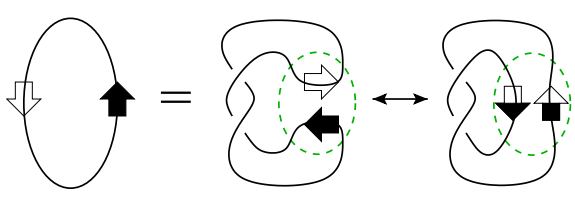

(A) Direct repeats

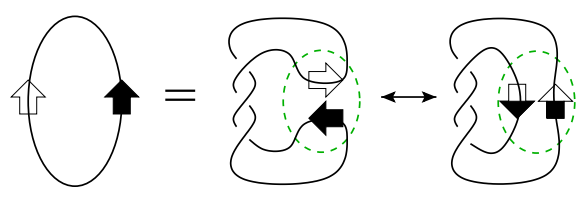

(B) Inverted repeats

Figure 6. (A) Recombination on directly repeated sites. (B) Recombination on inversely repeated sites.

$P$ with the tangle $R$ (Figure 3). By taking a very small tangle ball around the DNA segments which are broken by the recombinase, we can assume the tangle $P$ does not contain any crossings. Thus we can take the $P$ to be the 0 tangle. The remaining DNA configuration represented by the tangle $U$ is unchanged by the recombinase action (Figure 3).

Currently the local action of various recombinases have been modeled by $\left(0, \frac{1}{w}\right)$ moves where $|w|=0,1,2$ (for example, see Crisona, Weinberg, Peter, Sumners and Cozzarelli [5], Hodgman, Griffiths and Summers [18] and Sumners, Ernst, Spengler and Cozzarelli [30]). Thus the local action of a recombinase is believed to be equivalent to a band surgery. Moreover in some cases, the band surgery can be assumed to be coherent. When the sites are inversely repeated, recombination normally results in the inversion of one DNA segment with respect to the other one. Thus this case does not correspond to a coherent band surgery. However, when the sites are directly repeated or on different components, we can use these sites to orient the DNA. In this case, the chemistry of the reaction normally requires that the recombination correspond to a coherent band surgery.

\subsection{Xer recombination}

When acting on circular DNA, recombinases can invert a DNA segment, delete a DNA segment or fuse together two DNA circles (Figure 6). When two identical DNA circles fuse together into one larger DNA circle, the larger DNA circle contains two copies of the same DNA sequence (one from each of the identical circles). The larger DNA circle made up of two copies of the same DNA sequence is called a dimer $(\mathrm{di}=\mathrm{two}$, mer $=$ part). This can occur to the genome of the bacteria $E$. coli. When two circular genomes of $E$. coli fuse to form one larger circle containing two copies of the $E$. coli genome, the genome of the E. coli may not properly segregate when the host cell divides to form two new daughter cells. Thus for proper segregation of its genome upon cell division, E. coli needs a method to change the dimer into two monomers. This is the job of Xer recombinase. 
The biochemistry of Xer recombination is very similar to recombinases such as Flp and Cre. However its topology is very different. Cre and Flp can produce a spectrum of knots and links when acting on circular DNA. They can invert, delete, or fuse together DNA. Xer on the other hand produces a unique product dependent only on the starting configuration of the DNA (usually unknotted, but not always as seen below). Xer's function is to create two DNA circles from a larger DNA circle. In other words, its function is to performs deletions. Xer recombinase does not need to invert a DNA segment, and it should not accidently fuse two DNA circles into a dimer. But how does a protein know when it is acting on two segments from the same or different molecules or whether it is deleting or inverting a DNA segment? Xer uses a topological filter to ensure that it only performs deletions when acting on unknotted circular DNA (see Colloms, Bath and Sherratt [3]). The term topological filter is used by biologists to describe the mechanism in which a protein such as Xer sets up a specific protein-DNA topology in order to select a particular reaction pathway - in this case deletion instead of fusion or inversion.

When acting on unknotted circular DNA, Xer produces the $(2,4)$-torus link with linking number -2 (see [3]). It is believed that Xer uses the topological mechanism shown in Figure 7. The local action of Xer recombination is modeled by the small dashed circles: the $P=0$ tangle is changed into the $R=-1$ tangle. The larger black circles in Figure 7 denote the tangles modeling the entire protein-DNA complex. We will use $B$ and $E$ to represent these larger tangles modeling the protein-bound DNA before and after recombination, respectively. Xer uses accessory proteins to trap three DNA crossings. Thus this protein-DNA complex includes the Xer proteins, the accessory proteins and the three DNA crossings. It is modeled by the $B=-\frac{1}{3}$ tangle. If the Xer binding sites are directly repeated, the three crossings brings the Xer sites into proper conformation so that recombination can occur. Thus the protein bound DNA configuration changes from the $B=-\frac{1}{3}$ tangle to the $E=-\frac{4}{3}$ tangle. We would need to change the orientation of one of the arrows in Figure 7 if the DNA sites were inversely repeated. Xer cannot act on such a conformation and thus inversion is prevented. The $B=-\frac{1}{3}$ tangle conformation also makes fusion of two unlinked DNA circles unlikely as it is biologically difficult to form the three crossings in the $B=-\frac{1}{3}$ tangle if the DNA comes from two unlinked circular DNA molecules. Thus deletion is normally preferred over both inversion and fusion.

\subsection{Xer recombination from $(2,2 k)$-torus link to $2 k+1$ crossing knot}

In [1] Bath, Sherratt and Colloms studied Xer site-specific recombination on DNA catenanes whose link types were $(2,2 k)$-torus links, $(k \geq 2)$. Xer binding sites were placed on each component of these links, and these sites were used to orient the 


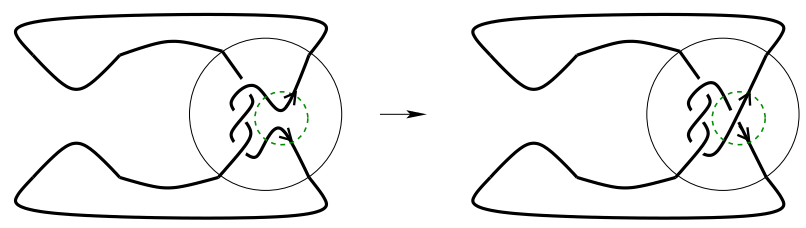

Figure 7. Xer recombination on the unknot results in the $(2,4)$-torus link

$(2,2 k)$-torus link substrates. If these $(2,2 k)$-torus links had linking number $-k$ and if $k \geq 3$, then Xer recombination can occur relatively efficiently and yields DNA knots with $2 k+1$ crossings. That is, Xer recombination can result in the fusion of two DNA molecules. For example, Figure 8 shows possible configurations of DNA before and after recombination in the case where $k=3$. Xer did not act efficiently when the $(2,2 k)$-torus links had linking number $k$, nor did it act efficiently on $N(4)$ regardless of the orientation of the Xer binding sites. Recall that the topological filter used by Xer is suppose to prevent fusion from occurring. Thus in order to understand why Xer can perform fusion on some substrates but not others, we need to understand the topology of these reactions. According to Theorem 3.1, assuming Xer's local action corresponds to a coherent band surgery, it is not mathematically possible for Xer to act on a $(2,2 k)$-torus links with linking number $k \geq 3$ and produce the knot $N\left(\frac{4 m n-1}{2 m}\right)$. This corresponds nicely with Xer's inability to act when the $(2,2 k)$-torus links had linking number $k, k \geq 2$. Theorem 4.1 only applies when the product is $N\left(\frac{4 m n-1}{2 m}\right)$ and does not give us any information about other types of knots, but it is a good first step. That Xer can act on $(2,2 k)$-torus links with linking number $-k$ when $k \geq 3$, but not on the $(2,4)$ torus link is more intriguing.

To better understand Xer recombination, we will first focus on its local action. By Theorem 2.1, a $\left(0, \frac{1}{w}\right)$-move and $\left(0, \frac{1}{w^{\prime}}\right)$-move are equivalent to each other for any integers $w$ and $w^{\prime}$. The local action of Xer recombination has been modeled by replacing the $P=0$-tangle with the $R=-1$-tangle. Thus we fix the integer $w$ : $w=-1$ in Theorem 4.1 and corollaries. Recall that the chemistry of the reaction normally requires that the local action correspond to a coherent band surgery.

Theorem 4.1 Suppose $N(U+0)=N(2 k)$ and $N(U+(-1))=N\left(\frac{4 m n-1}{2 m}\right)$, where $k \geq 1$ and $N\left(\frac{4 m n-1}{2 m}\right)$ has $2 k+1$ crossings. Then $m n>0,|m+n|=k+1$, and $U=\left(\frac{-1}{2 m-1}+\frac{-1}{2 n-1}\right)$ or $\left(\frac{-1}{2 n-1}+\frac{-1}{2 m-1}\right)$ (see Figure 10).

Proof First we determine the crossing number of the knot $N\left(\frac{4 m n-1}{2 m}\right) . N\left(\frac{4 m n-1}{2 m}\right)=$ $N\left(2 n+\frac{1}{-2 m}\right)$. Thus if $m n<0$, the crossing number of the knot $N\left(\frac{4 m n-1}{2 m}\right)$ is $2|m+n|$ since a reduced alternating diagram gives the crossing number of the corresponding 


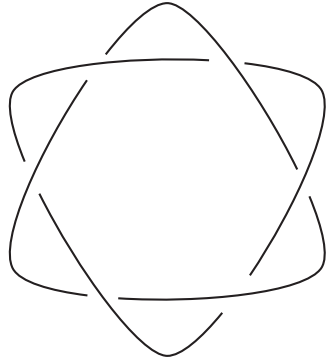

$(2,6)$-torus link

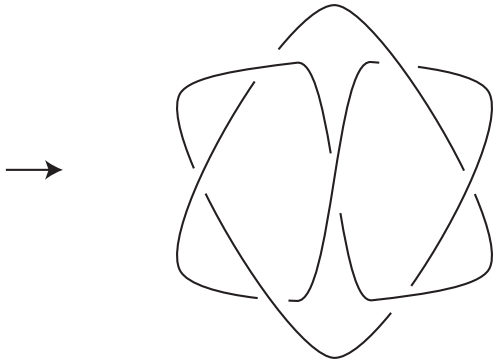

74

Figure 8. Xer recombination from the $(2,6)$-torus link to the $7_{4}$ knot
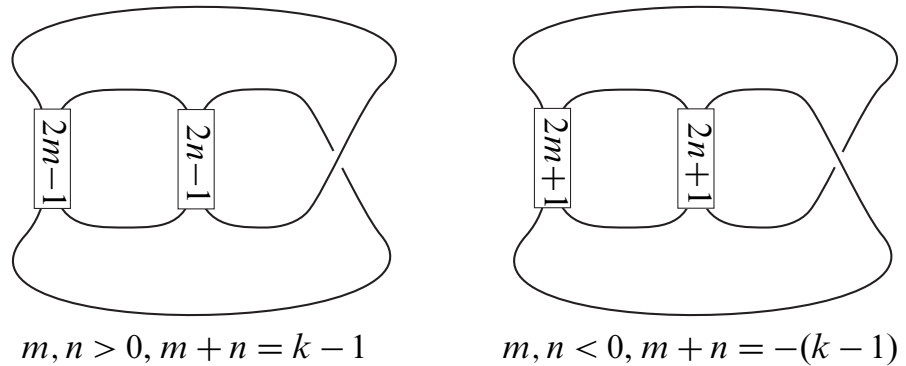

Figure 9. Knots with $2 k+1$ crossings

knot. Hence $m n>0$. Note that

$N\left(\frac{-1}{2 m-1}+\frac{-1}{2 n-1}+(-1)\right)=N\left(\frac{-1}{2 m-1}+\frac{-2 n}{2 n-1}\right)=N\left(\frac{-(2 n-1)-2 n(2 m-1)}{-2 m}\right)=N\left(\frac{4 m n-1}{2 m}\right)$.

Also

$N\left(\frac{-1}{2 m+1}+\frac{-1}{2 n+1}+1\right)=N\left(\frac{-1}{2 m+1}+\frac{2 n}{2 n+1}\right)=N\left(\frac{-(2 n+1)+2 n(2 m+1)}{2 m}\right)=N\left(\frac{4 m n-1}{2 m}\right)$.

Thus the knot $N\left(\frac{4 m n-1}{2 m}\right)$ is ambient isotopic to the pretzel knots $P(2 m-1,2 n-1,1)$ and $P(2 m+1,2 n+1,-1)$. Thus the crossing number of a knot $N\left(\frac{4 m n-1}{2 m}\right)$ is $2|m+n|-1$ if $m n>0$. Hence the crossing number of $N\left(\frac{4 m n-1}{2 m}\right)$ is $2 k+1$ if and only if $m n>0$ and $|m+n|=k+1$, see Figure 9 .

Applying Theorem 3.1 for

$$
\begin{aligned}
N((U+(-1))+0) & =N(U+(-1))=N\left(\frac{4 m n-1}{2 m}\right) \\
\text { and } \quad N((U+(-1))+1) & =N(U+0)=N(2 k)
\end{aligned}
$$


together with an assumption $m n>0,|m+n|=k+1 \geq 4, w=1$, we obtain from case (4) $k=m+n-1$, that

$$
(U+(-1))=\left\{\begin{array}{l}
\left(\frac{-1}{2 m-1}+\frac{-1}{2 n-1}\right) \circ(-1)=\left(\frac{-1}{2 m-1}+\frac{-1}{2 n-1}\right)+(-1) \quad \text { or } \\
\left(\frac{-1}{2 n-1}+\frac{-1}{2 m-1}\right) \circ(-1)=\left(\frac{-1}{2 n-1}+\frac{-1}{2 m-1}\right)+(-1),
\end{array}\right.
$$

and so

$$
U= \begin{cases}\left(\frac{-1}{2 m-1}+\frac{-1}{2 n-1}\right) & \text { or } \\ \left(\frac{-1}{2 n-1}+\frac{-1}{2 m-1}\right) & \end{cases}
$$

see Figure 10. When $m n>0$ and $|m+n|=k+1$, case (3) does not occur and cases (1) and (2) are included in case (4).

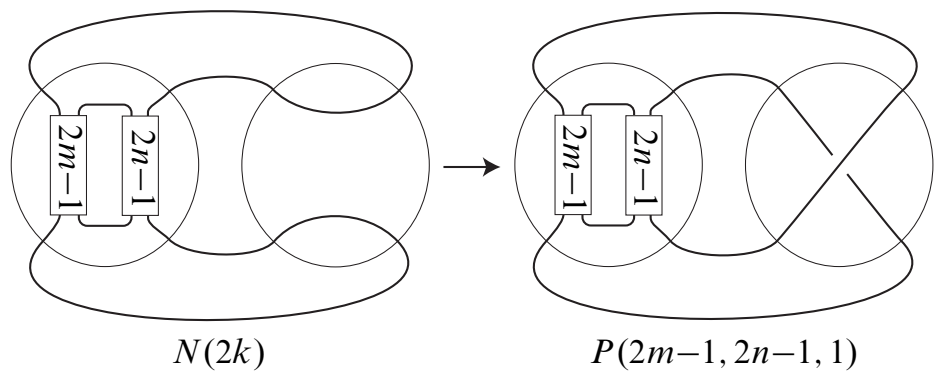

Figure 10. Xer recombination from $N(2 k)$ to $P(2 m-1,2 n-1,1)$

By Theorem 2.1, a $(0,-1)-$ move is equivalent to a $\left(0, \frac{1}{w}\right)-$ move, because $\frac{1}{w}=\frac{1}{-1-h}$ for an integer $h(h=-w-1)$. Then we obtain Corollary 4.2 below from Theorem 4.1.

Corollary 4.2 Suppose $N(U+0)=N(2 k)$ and $N\left(U+\frac{1}{w}\right)=N\left(\frac{4 m n-1}{2 m}\right)$, where $k \geq 1$ and $N\left(\frac{4 m n-1}{2 m}\right)$ has $2 k+1$ crossings. Then $m n>0,|m+n|=k+1$, and $U=\left(\frac{-1}{2 m-1}+\frac{-1}{2 n-1}\right) \circ(-w-1,0)$ or $\left(\frac{-1}{2 n-1}+\frac{-1}{2 m-1}\right) \circ(-w-1,0)$.

In the following corollaries, we apply Theorem 4.1 to the cases involving $(2,2 k)$-torus links with linking number $-k$ where $k=3,4,5$.

Corollary 4.3 Suppose $N(U+0)=N(6)$.

(1) If $N(U+(-1))=7_{2}=N\left( \pm \frac{11}{2}\right)$, then $U=\left(-\frac{6}{5}\right)$, so $N(U+(-1))=N\left(\frac{11}{2}\right)$.

(2) If $N(U+(-1))=74=N\left( \pm \frac{15}{4}\right)$, then $U=\left(\frac{-1}{3}+\frac{-1}{3}\right)$, so $N(U+(-1))=N\left(\frac{15}{4}\right)$.

Corollary 4.4 Suppose $N(U+0)=N(8)$.

(1) If $N(U+(-1))=9_{2}=N\left( \pm \frac{15}{2}\right)$, then $U=\left(-\frac{8}{7}\right)$, so $N(U+(-1))=N\left(\frac{15}{2}\right)$. 
(2) If $N(U+(-1))=9_{5}=N\left( \pm \frac{23}{4}\right)$, then $U=\left(\frac{-1}{3}+\frac{-1}{5}\right)$ or $U=\left(\frac{-1}{5}+\frac{-1}{3}\right)$, so $N(U+(-1))=N\left(\frac{23}{4}\right)$.

\section{Corollary 4.5 Suppose $N(U+0)=N(10)$.}

(1) If $N(U+(-1))=11 a 247=N\left( \pm \frac{19}{2}\right)$, then $U=\left(-\frac{10}{9}\right)$, so $N(U+(-1))=N\left(\frac{19}{2}\right)$.

(2) If $N(U+(-1))=11 a 343=N\left( \pm \frac{31}{4}\right)$, then $U=\left(\frac{-1}{3}+\frac{-1}{7}\right)$ or $U=\left(\frac{-1}{7}+\frac{-1}{3}\right)$, so $N(U+(-1))=N\left(\frac{31}{4}\right)$.

(3) If $N(U+(-1))=11 a 363=N\left( \pm \frac{35}{6}\right)$, then $U=\left(\frac{-1}{5}+\frac{-1}{5}\right)$, so $N(U+(-1))=$ $N\left(\frac{35}{6}\right)$.

\subsection{Xer recombination from trefoil knot to Hopf link}

Our original description of Xer recombination was a simple one. So far we have only described one type of Xer action. The action of proteins depends on reaction conditions. For Xer, its action will be affected by both accessory proteins and the sequence to which it binds. Xer is a site-specific recombinase (meaning it acts on specific DNA sequences). But there are several different specific sequences upon which it can act. Previously we described the experiment in which $(2,2 k)$ torus links were converted into knots with $2 k+1$ crossings. This case involved the proteins XerC, XerD, and PepA acting on the DNA sequence psi. In this case in order for Xer to act, these proteins trapped three DNA crossings (Figure 7).

We will now describe a second set-up for Xer recombination. The proteins XerC, $\mathrm{XerD}$, and FtsK can also act on the E. coli dif sequence. To distinguish this second type of action we will refer to this as XerCD-dif-FtsK recombination. This system can resolve dimers. It can also unlink DNA links in vitro, that is, in a test tube (see Ip, Bregu, Barre and Sherratt [19]), and in vivo, that is, in the cell (see Grainge, Bregu, Vazquez, Sivanathan, Ip and Sherratt [15]). For XerCD-dif-FtsK recombination, the topology of the protein-bound DNA is much simpler. It is believed that FtsK is responsible for setting up a much simpler protein-bound DNA topology in which there is a projection where no DNA crossings are trapped. For a movie of a proposed model, see supplementary data in [19].

XerCD-dif-FtsK can unlink the $(2,2 k)$-torus link with linking number $\pm k$. When the linking number is $-k$, the observed products were the unknot and the unlink of two componts and the proposed pathway is believed to be $(2,2 k)$-torus link $\rightarrow$ unknot $\rightarrow$ unlink [19]. The related tangle equations are easily solved using Hirasawa and Shimokawa [17] and TopoIce-R [8]. When the linking number is $k$, a stepwise unlinking model was proposed [19] in which each round of recombination reduces the 
crossing number by one: the pathway is the $(2,2 k)$-torus link, the $(2,2 k-1)$-torus knot, the $(2,2 k-2)$-torus link, $\ldots$, the $(2,4)$-torus link, the trefoil knot, the Hopf link, the trivial knot, the trivial link. See Shimokawa, Ishihara and Vazquez [29] for a mathematical study. If we assume each recombination is modeled by a coherent band surgery, the following corollary of Theorem 3.1 characterizes the band surgery between the trefoil knot and the Hopf link. The band surgery between the Hopf link and the trivial knot is characterized by Thompson [31] and Hirasawa and Shimokawa [17] and the band surgery between the trivial knot and the trivial link is characterized by Scharlemann [27].

Corollary 4.6 If $N(U+0)=N(3)$ (trefoil knot) and $N\left(U+\frac{1}{w}\right)=N(2)$ (Hopf link), then $U=\left(\frac{3}{-3 w-2}\right)$. If $N(U+0)=N(3)$ and $N(U+(-1))=N(2)$, then $U=(3)$ (see Figure 11).

Proof We apply Theorem 3.1 by putting $m=n=k=w=-1$. Then we obtained the solutions (1), (2) and (3). Each of them shows $U=(3)$.

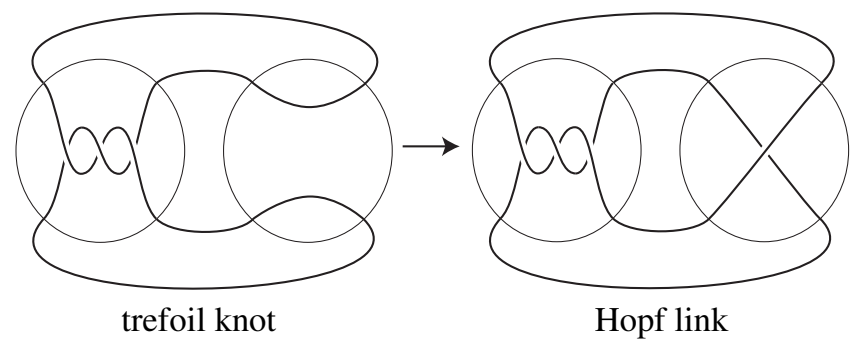

Figure 11. Xer recombination from the trefoil knot to the Hopf link

\subsection{A comment on converting $N(4)$ into the trefoil knot}

In the remark below, we look at one solution for converting $N(4)$ into $N(3)$.

Remark 4.7 If $U=(4)$, then $N(U+0)=N(4)$ where $N(4)$ has linking number +2 and $N(U+(-1))=N(3)$.

If $N(4)$ has linking number +2 , we cannot apply Theorem 3.1. In this case the coherent band corresponding to the rational tangle surgery can be isotoped to lie in the genus one Seifert surface of $N(4)$, but not in the minimal Seifert surface of $N(3)$ (See Theorem 5.2). 


\section{Proof of Theorem 3.1}

In this section we prove Theorem 3.1 when $m, n \neq 0$. Thus we wish to solve the system of equations $N(U+0)=N\left(\frac{4 m n-1}{2 m}\right)$ and $N\left(U+\frac{1}{w}\right)=(2,2 k)$-torus link. We will use that this rational tangle surgery corresponds to a band surgery. We assume the band surgery is coherent. For a coherent band surgery, the relation between the signatures of $L$ and $L_{b}$ is known.

Theorem 5.1 (Murasugi [24, Lemma 7.1]) If $L_{b}$ is obtained from $L$ by a coherent band surgery, then $\left|\sigma(L)-\sigma\left(L_{b}\right)\right| \leq 1$.

We consider the case where $L$ is a $2-$ bridge knot $N\left(\frac{4 m n-1}{2 m}\right)$ and $L_{b}$ is $N(2 k)$. Suppose $N(2 k)$ has linking number $k$ where $|k|>2$. Then the absolute value of its signature is $2|k|-1$ while $\left|\sigma\left(N\left(\frac{4 m n-1}{2 m}\right)\right)\right|=0$ or 2 . Thus when $|k|>2$, the system of equations, $N(U+0)=N\left(\frac{4 m n-1}{2 m}\right)$ and $N\left(U+\frac{1}{w}\right)=N(2 k)$ where $N(2 k)$ has linking number $k$ has no solution when the rational tangle surgery corresponds to a coherent band surgery. Since $N(2)=N(-2)$ as unoriented links, we can assume that the Hopf link, $N(2 k)$ has linking number $-k$ where $k= \pm 1$. Hence from here on, we will assume $N(2 k)$ has linking number $-k$.
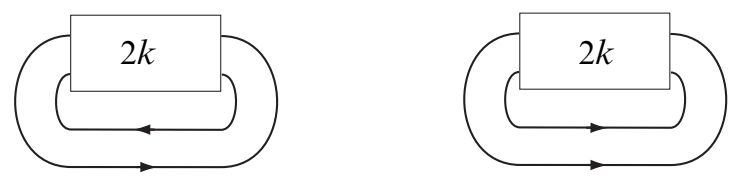

Figure 12. $(2,2 k)$-torus links: the left diagram has linking number $-k$ while the right diagram has linking number $k$.

Theorem 5.2 (Deruelle, Miyazaki and Motegi [10], Hirasawa and Shimokawa [17], Scharlemann and Thompson [28]) Suppose that $b$ is a band of a coherent band surgery from $L$ to $L_{b}$. Then $\chi(L) \leq \chi\left(L_{b}\right)-1$ if and only if $L$ has a taut Seifert surface containing $b$.

Since the Seifert surface for $L$ is connected, it is taut if and only if it is of minimal genus.

In our case $\chi(L)=-1, \chi\left(L_{b}\right)=2$ if $L_{b}$ is the unlink of two components, and $\chi\left(L_{b}\right)=0$ otherwise. The link $L$ is a genus one 2-bridge knot and genus one Seifert surfaces of $L$ are characterized by Hatcher and Thurston [16]; there are only two Seifert surfaces of $L$ up to equivalence as shown in Figure 13. Here two Seifert surfaces for an oriented link are said to be equivalent if there exists an ambient isotopy such that 
one Seifert surface is moved to the other by the isotopy and the link is fixed as a set throughout the isotopy. The surfaces $S_{1}$ and $S_{2}$ in Figure 13 are genus one Seifert surfaces of $L$ which are obtained by plumbing of two annuli with $m$ and $n$ full twists. Moreover, by Kobayashi [20] it is known that $S_{1}$ is equivalent to $S_{2}$ if and only if $m= \pm 1$ or $n= \pm 1$. Let $S$ be a minimal Seifert surface of $L$ which contains $b$ (that is, $S=S_{1}$ or $S_{2}$ ). The Seifert surface $S_{1}$ can be isotoped to $S_{2}$ via a $\pi$ rotation which moves $L$, but we will prove results for the stronger definition of equivalence given above.
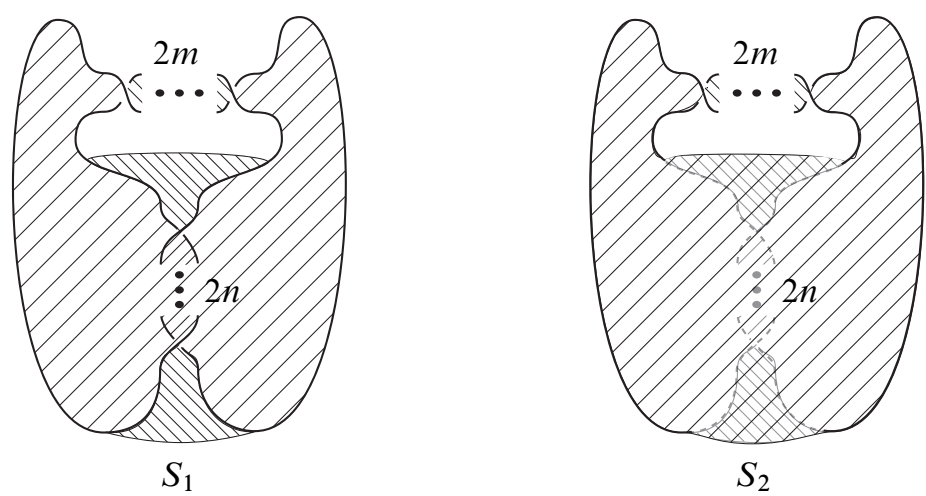

Figure 13. Minimal genus Seifert surfaces for $N\left(\frac{4 m n-1}{2 m}\right)$

We will give a parametrization of bands attached to $L$ which are contained in $S$. If $S-b$ is not connected, then the core of the band is boundary parallel. Thus $L_{b}$ is a split link with $L$ and the unknot. This does not satisfy our hypothesis since $L$ is not the unknot and $L_{b}=N(2 k)$. If $b$ is contained in $S$ and if $S-b$ is connected, then $\mathrm{Cl}(S-b)$ is an annulus in $S^{3}$. Let $\gamma_{b}$ denote a core of the annulus. Note that the boundary of this annulus is exactly $L_{b}$, so $L_{b}=N(2 k)$ if and only if $\gamma_{b}$ is a trivial knot. A band $b$ determines a unique knot $\gamma_{b}$ (up to isotopy in $S$ ). Conversely, given a knot in $S$, there exists a unique band in $S$ (up to isotopy in $S$ ) which is disjoint from that knot. Thus there exists a one to one correspondence between ambient isotopy classes of bands $b$ in $S$ and ambient isotopy classes of unoriented knots $\gamma_{b}$ in $S$. In order to parametrize a band $b$ in $S$, we will parametrize such a knot $\gamma_{b}$ in $S$. Recall that $S$ is a plumbing of two annuli with $m$ and $n$ full twists. Let $c_{M}$ (resp. $c_{N}$ ) be the oriented unknot shown in Figure 14 which spans a disk that transversely meets the annulus with $m$ (resp. $n$ ) full twists in an arc. For parametrization, we give an orientation of $\gamma_{b}$. Let $p=\operatorname{lk}\left(\gamma_{b}, c_{M}\right)$ and $q=\operatorname{lk}\left(\gamma_{b}, c_{N}\right)$. Then $\gamma_{b}$ is parametrized by $\pm(p, q)$. 


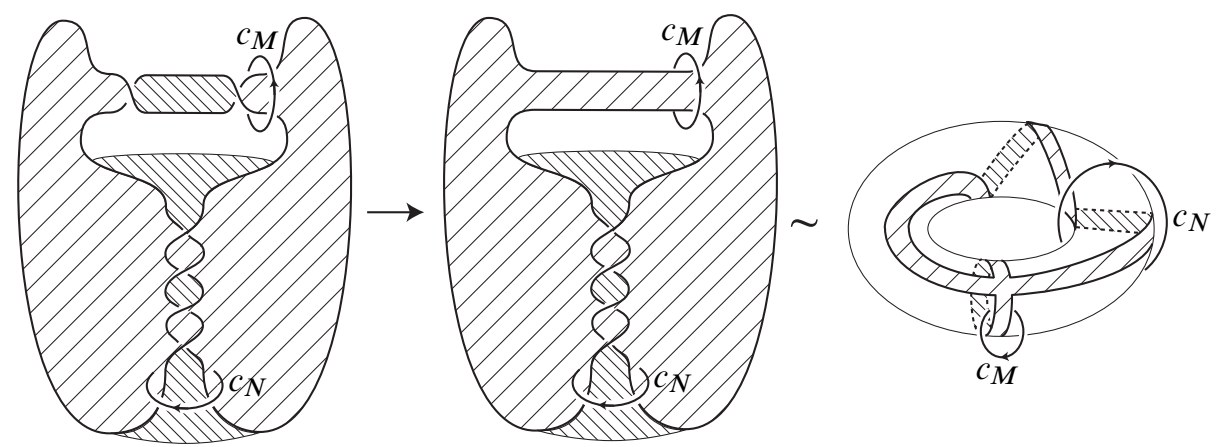

Figure 14. (-m)-twist along $c_{M}$

We consider a $(-m)$-twist along $c_{M}$. Then after the twist, $S$ lies on a standard torus in $S^{3}$ as shown in Figure 14. Hence $\gamma_{b}$ becomes a torus knot after the twist. Motegi [23] characterized twists on an unknot which yield torus knots.

Theorem 5.3 (Motegi [23, Theorem 3.8]) Suppose a knot $K_{-m}$, which is obtained from a trivial knot $K$ by a $(-m)-t$ wist along a trivial $k n o t ~ C$, is a torus knot. Then, except for trivial examples, $m= \pm 1$.

Trivial examples are shown in Figure 15. In trivial examples, $C$ is ambient isotopic (in $S^{3}-K$ ) to the core loop of a solid torus whose boundary torus contains $K$ and $K_{-m}$.

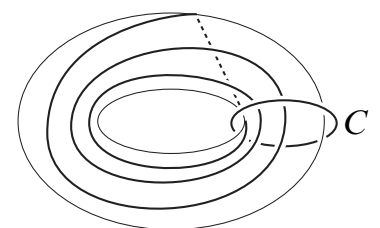

$(1, p)$-torus knot

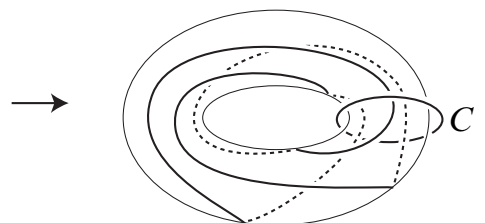

$(1-m p, p)$-torus knot

Figure 15. Trivial example: A $(1-m p, p)$-torus knot is obtained from a $(1, p)$-torus knot, which is a trivial knot, by $(-m)$-twist along a trivial knot $C$.

By applying Theorem 5.3 for $C=c_{M}$ and $K=\gamma_{b}$, we obtain the following lemma.

Lemma 5.4 If $\gamma_{b}$ is a trivial knot, then one of the following holds.

(1) $|p| \leq 1$ and $|q| \leq 1$. Namely $(p, q)= \pm(1,0), \pm(0,1), \pm(1,1)$ or $\pm(1,-1)$.

(2) $n=1$ and $(p, q)= \pm(1,-2)$.

(3) $n=-1$ and $(p, q)= \pm(1,2)$. 
(4) $m= \pm 1$.

Remark 5.5 If (1) of Lemma 5.4 holds, then for any $m$ and $n, \gamma_{b}$ is trivial. If (2) or (3) holds, then for any $m, \gamma_{b}$ is trivial.

Proof of Lemma 5.4 Let $\gamma_{b}^{\prime}$ be a knot which is obtained from $\gamma_{b}$ by $(-m)$-twist along $c_{M}$. The standard torus on which $\gamma_{b}^{\prime}$ lies divides $S^{3}$ into the union of two solid tori. The loop $c_{N}$ maps to the core knot for one of these tori. Let $c$ be the core knot for the other torus as in Figure 16.

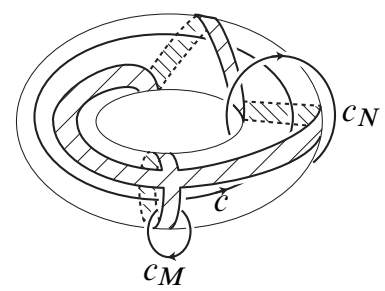

Figure 16

Then linking numbers can be calculated as follows.

$$
\begin{aligned}
\operatorname{lk}\left(\gamma_{b}^{\prime}, c_{M}\right) & =\operatorname{lk}\left(\gamma_{b}, c_{M}\right)=p, \\
\operatorname{lk}\left(\gamma_{b}^{\prime}, c_{N}\right) & =\operatorname{lk}\left(\gamma_{b}, c_{N}\right)=q, \\
\operatorname{lk}\left(\gamma_{b}^{\prime}, c\right) & =p+n q .
\end{aligned}
$$

By applying Theorem 5.3 for $C=c_{M}, K=\gamma_{b}, K_{-m}=\gamma_{b}^{\prime}$, except trivial examples, we have the conclusion (4) of Lemma 5.4. Thus it is enough to consider the trivial examples. Since a $(-m)$-twist along $C_{M}$ represents a trivial example, we have

$$
\gamma_{b}^{\prime}=( \pm 1-m p, p) \text {-torus knot. }
$$

On the other hand, from the linking numbers of $\gamma_{b}^{\prime}$ with $c_{N}$ and with $c$ above, we have

$$
\gamma_{b}^{\prime}=(q, p+n q) \text {-torus knot. }
$$

Suppose $\gamma_{b}^{\prime}$ is the trivial knot. From equation (1), $|p|=1$ or $| \pm 1-m p|=1$. From equation (2), $|q|=1$ or $|p+n q|=1$. If $|p|=|q|=1$, then the conclusion (1) of Lemma 5.4 holds. If $|p|=|p+n q|=1$, then $|n q|=1 \pm|p|=0$ or 2 , so one of the conclusions (1), (2) and (3) of Lemma 5.4 holds. If $|p| \neq 1$ and $| \pm 1-m p|=1$, then $|m p| \leq 2$, so $(p, q)= \pm(0,1)$ or $m= \pm 1$, that is, the conclusion (1) or (4) of Lemma 5.4 holds. 
Suppose $\gamma_{b}^{\prime}$ is a non-trivial torus knot. From equation (1), $|p| \geq 2$. From equation (2), $|q| \geq 2$. The integers $p$ and $q$ are relatively prime, so $|p| \neq|q|$. By contrasting equations (1) and (2), $( \pm 1-m p, p)= \pm(q, p+n q)$. Since $n q \neq 0$,

$$
\begin{aligned}
\pm 1-m p & =-q, \\
\text { and } \quad p & =-(p+n q) .
\end{aligned}
$$

If $|q|<|p|$, by equation (3), $|q|<|p| \leq|m p|=|q \pm 1| \leq|q|+1$. then $|p|=|m p|$, so the conclusion (4) of Lemma 5.4 holds. If $|q|>|p|$, by equation (4), $2|p|=|n q|>|n p|$, then $|n|=1$ and $2|p|=|q|$. Since integers $p$ and $q$ are relatively prime and $|p| \geq 2$, it does not happen.

Next we state a well known lemma of a braid presentation for a trivial knot (see, for example, Morton and Hadji [22]).

Lemma 5.6 If a trivial knot has a positive or negative $n$-braid presentation with $m$ crossings, then $m=n-1$.

We say that two bands attached to a link $L$ are equivalent with respect to $L$ if there exists an ambient isotopy of $S$ in $S^{3}$ such that one band is moved to the other by the isotopy and $L$ is fixed as a set throughout the isotopy. Now we state the main theorem of this section.

Theorem 5.7 Let $L$ be a 2-bridge knot $N\left(\frac{4 m n-1}{2 m}\right)$ in $S^{3}$ with $m, n \neq 0$. Suppose that $b$ is a band of a coherent band surgery from $L$ to $L_{b}$, and $L_{b}$ is a 2-bridge link $N(2 k)$ with linking number $-k$. Then the band $b$ is equivalent to one of the six bands $b_{1}, b_{2}, b_{3}, b_{4}, b_{5}$ and $b_{6}$ in Figure 17 with respect to $L$.

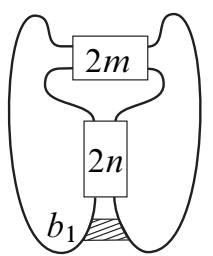

$k=m$

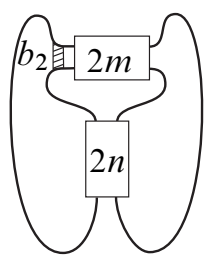

$k=n$

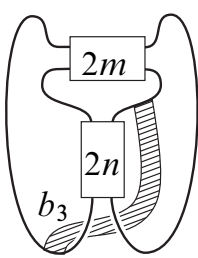

$k=m+n+1$
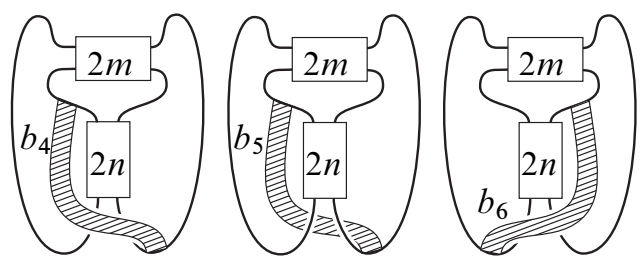

$k=m+n+1$ $k=m+n-1 \quad k=m+n-1$

Figure 17

Remark 5.8 (1) The band for $(p, q)= \pm(1,0)$ and $S=S_{1}$ or $S_{2}$ corresponds to $b_{1}$.

(2) The band for $(p, q)= \pm(0,1)$ and $S=S_{1}$ or $S_{2}$ corresponds to $b_{2}$. 
(3) The band for $(p, q)= \pm(1,1)$ and $S=S_{1}$ (resp. $\left.S_{2}\right)$ corresponds to $b_{3}$ (resp. $\left.b_{4}\right)$.

(4) The band for $(p, q)= \pm(1,-1)$ and $S=S_{1}$ (resp. $\left.S_{2}\right)$ corresponds to $b_{5}$ (resp. $\left.b_{6}\right)$.

Remark 5.9 Suppose that $m= \pm 1$ or $n= \pm 1$. Then two Seifert surfaces $S_{1}$ and $S_{2}$ are equivalent (fixing $L$ ). By the ambient isotopy of this equivalence, we have the following: If $m=1$ (resp. $m=-1$ ), then the three bands $b_{2}, b_{5}, b_{6}$ (resp. $b_{2}, b_{3}, b_{4}$ ) are equivalent to each other with respect to $L$. If $n=1$ (resp. $n=-1$ ), then the three bands $b_{1}, b_{5}, b_{6}$ (resp. $b_{1}, b_{3}, b_{4}$ ) are equivalent to each other with respect to $L$. If $m=n=1$ (resp. $m=n=-1$ ) (that is, $L$ is the trefoil knot), then the four bands $b_{1}, b_{2}, b_{5}, b_{6}$ (resp. $b_{1}, b_{2}, b_{3}, b_{4}$ ) are equivalent to each other with respect to $L$, and the two bands $b_{3}, b_{4}$ (resp. $b_{5}, b_{6}$ ) are equivalent with respect to $L$. If $m=1, n=-1$ (resp. $m=-1, n=1$ ) (that is, $L$ is the figure eight knot), then the three bands $b_{1}, b_{3}, b_{4}$ (resp. $b_{1}, b_{5}, b_{6}$ ) are equivalent to each other with respect to $L$, and the three bands $b_{2}, b_{5}, b_{6}$ (resp. $b_{2}, b_{3}, b_{4}$ ) are equivalent to each other with respect to $L$.

Proof of Theorem 5.7 Let $L$ be a 2-bridge knot $N\left(\frac{4 m n-1}{2 m}\right)$ in $S^{3}$ with $m, n \neq$ 0 . Since $L$ is symmetric for $m$ and $n$, that is, $N\left(\frac{4 m n-1}{2 n}\right)$ is ambient isotopic to $N\left(\frac{4 m n-1}{2 m}\right)$, without loss of generality, we may assume that $|m| \geq|n| \geq 1$. Suppose, for a band $b, L_{b}$ is a 2 -bridge link $N(2 k)$ with linking number $-k$. We may assume that $b \subset S$ where $S$ is a minimal Seifert surface of $L$, and so $S=S_{1}$ or $S_{2}$ in Figure 13.

Suppose the conclusion (1) of Lemma 5.4 holds. Then the band is equivalent to one of $b_{1}, b_{2}, b_{3}, b_{4}, b_{5}$ and $b_{6}$ with respect to $L$, see Remark 5.8 .

Suppose the conclusion (2) of Lemma 5.4 holds. In this case $S_{1}$ and $S_{2}$ are equivalent. By the ambient isotopy of this equivalence, the band for $(p, q)= \pm(1,-2)$ and $S=S_{1}$ (resp. $S=S_{2}$ ) is equivalent to $b_{4}$ (resp. $b_{3}$ ) with respect to $L$.

Suppose the conclusion (3) of Lemma 5.4 holds. Similarly to above, the band for $(p, q)= \pm(1,2)$ and $S=S_{1}$ (resp. $\left.S=S_{2}\right)$ is equivalent to $b_{6}$ (resp. $\left.b_{5}\right)$ with respect to $L$.

From now on we will consider the remaining case where $|m|=|n|=1$. There are symmetries of $\gamma_{b}$ : that is, the parameters $(m, n, p, q)=(a, b, c, d),(b, a, d, c)$ and $(-a,-b, c,-d)$ determine the same knot type of $\gamma_{b}$ up to mirror image. Thus it is enough to consider the case where $p>q>0$. Then we obtain a $p$-string braid presentation for $\gamma_{b}$ by moving $S$ as shown in Figure 18. Using this braid presentations 

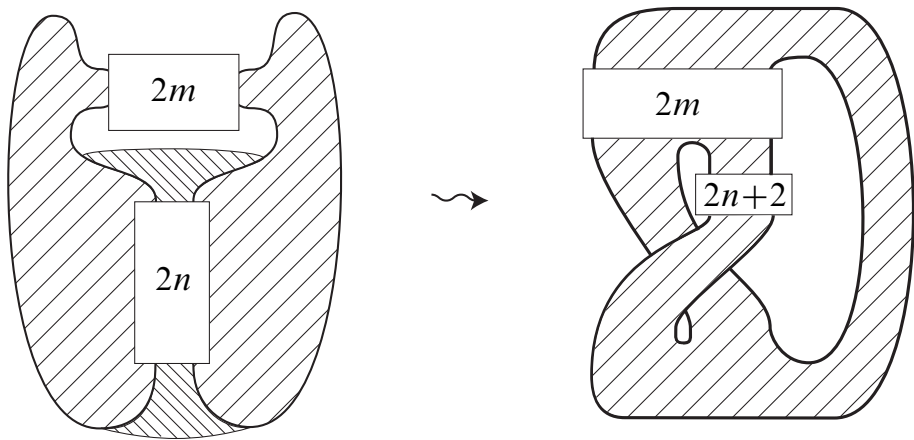

Figure 18. Recall that a positive (negative) number inside a box corresponds to right (left) handed half twists. For the figure on the left, the box containing $2 m$ represents $m$ horizontal twists. The remaining three boxes contain vertical twists.
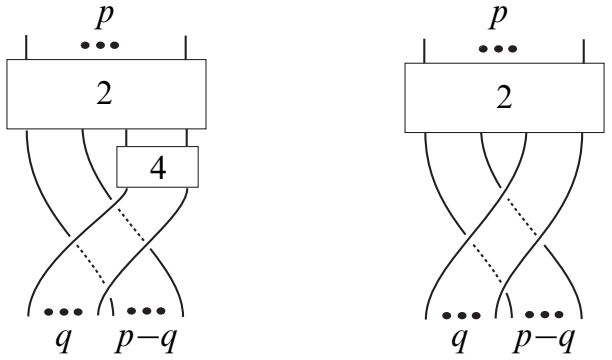

Figure 19. Left: $m=n=1$; Right: $m=1, n=-1$
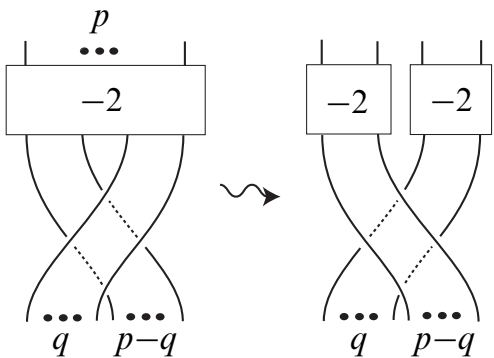

Figure 20. $m=n=-1, p>q>0$ : The $p$-string braid on the left simplifies via two right-handed half-twists to the negative braid on the right. Note that the right braid has $p-1$ crossings (and hence its closure is the unknot) if and only if $p=2$ and $q=1$.

for $\gamma_{b}$ and Lemma 5.6, we will decide whether or not the knot $\gamma_{b}$ is trivial. There are three cases for $m$ and $n$ : the first is where $m=1$ and $n= \pm 1$; the second is where $m=-1$ and $n=1$; and the third is where $m=n=-1$. In the first case, $\gamma_{b}$ has a positive braid presentation as shown in Figure 19, and hence $\gamma_{b}$ is a 

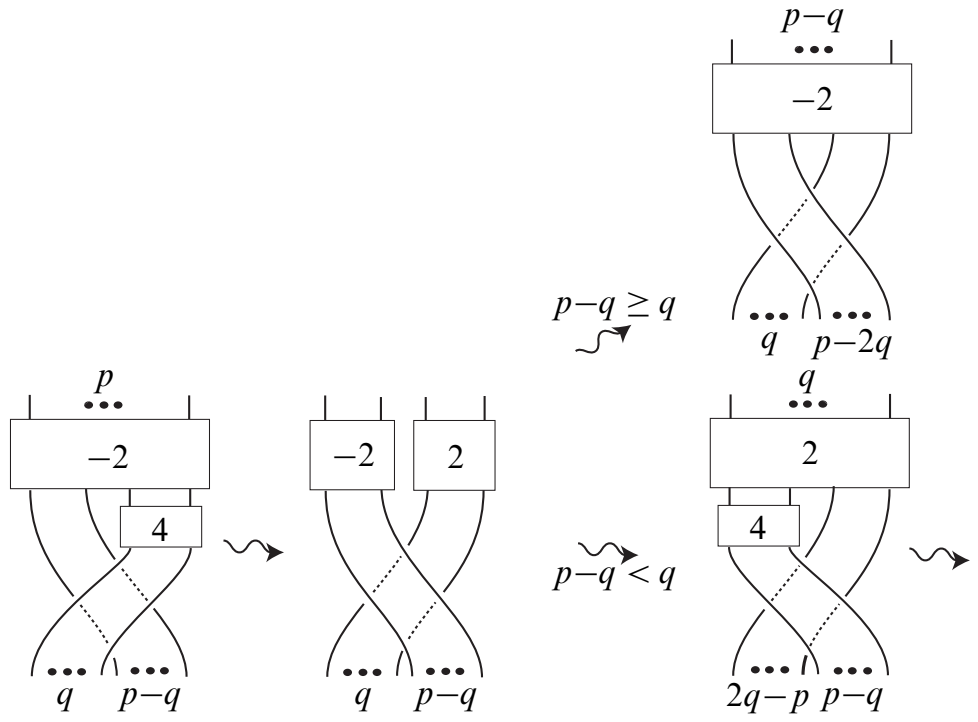

Figure 21. $m=-1, n=1, p>q>0$ : The braid on the left simplifies to one of the braids on the right. If $p-q \geq q$, then a $(p-q)-$ string negative braid is obtained (top right). This $(p-q)-$ string braid closes to the unknot if and only if $p-q=q=1$ If $p-q<q$, then a $q$-string braid is obtained which can be further simplified (bottom right). This $q$-string braid closes to the unknot if and only if $(p, q)=\left(F_{i+1}, F_{i}\right)$ where $F_{i}$ is the $i$ th Fibonacci number.

non-trivial knot. In the second case, by simplifying a braid presentation, $\gamma_{b}$ has a negative braid presentation as shown in Figure 20. Hence $\gamma_{b}$ is a non-trivial knot except when $(p, q)=(2,1)$. In the third case, we can simplify a braid presentation inductively if necessary as shown in Figure 21. Then $\gamma_{b}$ is a trivial knot if and only if $(p, q)=\left(F_{i+1}, F_{i}\right)$, where $i$ is any positive integer and $F_{i}$ is the $i$ th Fibonacci number; $F_{0}=0, F_{1}=1, F_{2}=1, F_{3}=2, F_{4}=3, F_{5}=5, \ldots$ By reconsidering the symmetries of $\gamma_{b}$, we can summarise a necessary and sufficient condition for a pair $(p, q)$ to determine a trivial knot $\gamma_{b}$ for $m=n=1$ and for $m=1, n=-1$ as follows.

Suppose that $m=n=1$, that is, $L$ is the trefoil knot. A knot $\gamma_{b}$ is trivial if and only if $(p, q)= \pm(0,1), \pm(1,0), \pm(1,1), \pm(1,-1), \pm(2,-1)$, or $\pm(1,-2)$. Now $L$ is a fibered knot and $S$ is a fibered surface. Let $\eta: S \rightarrow S$ be the monodromy map and let $\eta_{*}: H_{1}(S) \rightarrow H_{1}(S)$ be the homomorphism induced by $\eta$. We regard $(p, q)$ as an element of $H_{1}(S)$ and define two elements of $H_{1}(S)$ to be equivalent if $\eta_{*}^{k}$ maps one to the other for an integer $k$. Then $\pm(1,0), \pm(0,1) \pm(1,-1)$ belong to an equivalence class, and $\pm(1,1), \pm(2,-1), \pm(1,-2)$ belong to another one. This implies that the band is equivalent to $b_{1}$ or $b_{3}$ with respect to $L$, see Remark 5.8. 
Suppose that $m=1$ and $n=-1$, that is, $L$ is the figure eight knot. A knot $\gamma_{b}$ is trivial if and only if $(p, q)= \pm\left(F_{i}, F_{i+1}\right)$ or $\pm\left(F_{i+1},-F_{i}\right)$ for any non-negative integer $i$. Now $L$ is a fibered knot and $S$ is a fibered surface. Let $\eta: S \rightarrow S$ be the monodromy map and let $\eta^{*}: H_{1}(S) \rightarrow H_{1}(S)$ be the homomorphism induced by $\eta$. We regard $(p, q)$ as an element of $H_{1}(S)$ and define two elements of $H_{1}(S)$ to be equivalent if $\eta_{*}^{k}$ maps one to the other for an integer $k$. Then a set $A=$ $\left\{\left(F_{2 i-1},-F_{2 i-2}\right) \mid i \in \mathbb{N}\right\} \cup\left\{\left(F_{2 i-1}, F_{2 i}\right) \mid i \in \mathbb{N}\right\}$ is an equivalence class and a set $B=\left\{\left(-F_{2 i}, F_{2 i-1}\right) \mid i \in \mathbb{N}\right\} \cup\left\{\left(F_{2 i-2}, F_{2 i-1}\right) \mid i \in \mathbb{N}\right\}$ is another one. Note that the set $A$ contains $(1,0)$ and the set $B$ contains $(0,1)$. This implies that the band is equivalent to $b_{1}$ or $b_{2}$ with respect to $L$, see Remark 5.8. This completes the proof of Theorem 5.7.

In Figure 17, the two unions $L \cup b_{3}$ and $L \cup b_{4}$ (resp. $L \cup b_{5}$ and $L \cup b_{6}$ ) of a knot $L$ and bands are ambient isotopic to each other, and they are ambient isotopic to the third (resp. the fourth) of Figure 22. Then we obtain the following theorem.
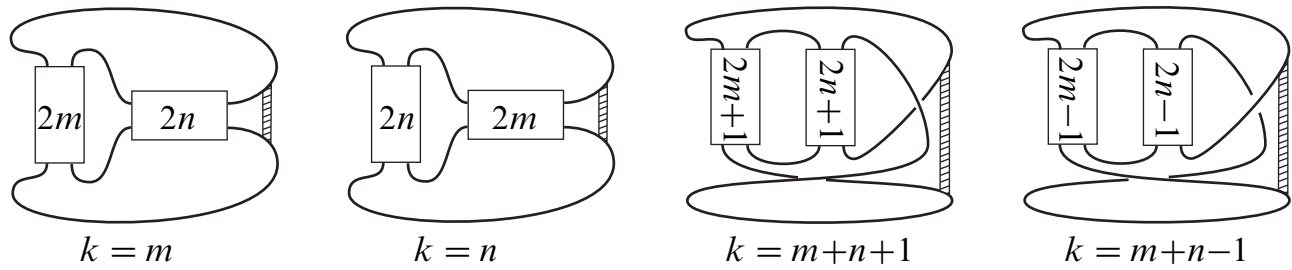

Figure 22

Theorem 5.10 Let $L$ be a $2-$ bridge knot $N\left(\frac{4 m n-1}{2 m}\right)$ in $S^{3}$ with $m, n \neq 0$. Suppose that $b$ is a band of a coherent band surgery from $L$ to $L_{b}$, and $L_{b}$ is a 2-bridge link $N(2 k)$ with linking number $-k$. Then the union $L \cup b$ of a knot $L$ and $a$ band $b$ is ambient isotopic to one of four in Figure 22.

Proof of Theorem 3.1 We consider a $\left(0, \frac{1}{w}\right)$ move as a band surgery as shown in Figure 4. Then we obtain Theorem 3.1 from Theorem 5.10 immediately.

\section{Non-band rational tangle surgery case}

Let $c(K)$ be the crossing number of the knot $K$. In this section we will characterize all non-band rational tangle surgeries on $N(2 k)$ yielding $N\left(\frac{4 m n-1}{2 m}\right)$, where $c(N(2 k))=$ $2 k$ and $c\left(N\left(\frac{4 m n-1}{2 m}\right)\right)=2 k+1$, for $k=3,4$ or 5 .

Suppose $N(U+0)=N(2 k)(=(2,2 k)$-torus link $)$, and $N\left(U+\frac{t}{w}\right)=$ a 2 -bridge knot $N\left(\frac{z}{v}\right)$. Per below the solutions to this system of equations are of the form 
$\frac{t}{w}=\frac{z-2 k v^{\prime}}{v^{\prime}-\left(z-2 k v^{\prime}\right) h}$ and $U=\left(\frac{2 k}{2 k h+1}\right)$, where $h$ is any integer and $v^{\prime}$ is an integer which satisfies $v^{\prime} \equiv v^{ \pm 1} \bmod z$, and so $N\left(\frac{z}{v^{\prime}}\right)=N\left(\frac{z}{v}\right)$. We show that there are no other solutions of the non-band rational tangle surgery for the cases where $\frac{z}{v}=\frac{4 m n-1}{2 m}$, $m n>0,|m+n|=k+1$ (which is the condition for $c\left(N\left(\frac{4 m n-1}{2 m}\right)\right)=2 k+1$, and $k=3,4$ or 5 .

Theorem 6.1 Suppose $N(U+0)=N(6)$ and $t \neq \pm 1$.

(1) If $N\left(U+\frac{t}{w}\right)=7_{2}\left(\frac{z}{v}= \pm \frac{11}{2}\right)$, then $\frac{t}{w}=\frac{11-6 v^{\prime}}{v^{\prime}-\left(11-6 v^{\prime}\right) h}$ and $U=\left(\frac{6}{6 h+1}\right)$, where $h$ is any integer and $v^{\prime}$ is an integer which satisfies $v^{\prime} \equiv \pm 2^{ \pm 1} \bmod 11$.

(2) If $N\left(U+\frac{t}{w}\right)=7_{4}\left(\frac{z}{v}= \pm \frac{15}{4}\right)$, then $\frac{t}{w}=\frac{15-6 v^{\prime}}{v^{\prime}-\left(15-6 v^{\prime}\right) h}$ and $U=\left(\frac{6}{6 h+1}\right)$, where $h$ is any integer and $v^{\prime}$ is an integer which satisfies $v^{\prime} \equiv \pm 4^{ \pm 1} \bmod 15$.

Theorem 6.2 Suppose $N(U+0)=N(8)$ and $t \neq \pm 1$.

(1) If $N\left(U+\frac{t}{w}\right)=9_{2}\left(\frac{z}{v}= \pm \frac{15}{2}\right)$, then $\frac{t}{w}=\frac{15-8 v^{\prime}}{v^{\prime}-\left(15-8 v^{\prime}\right) h}$ and $U=\left(\frac{8}{8 h+1}\right)$, where $h$ is any integer and $v^{\prime}$ is an integer which satisfies $v^{\prime} \equiv \pm 2^{ \pm 1} \bmod 15$.

(2) If $N\left(U+\frac{t}{w}\right)=9_{5}\left(\frac{z}{v}= \pm \frac{23}{4}\right)$, then $\frac{t}{w}=\frac{23-8 v^{\prime}}{v^{\prime}-\left(23-8 v^{\prime}\right) h}$ and $U=\left(\frac{8}{8 h+1}\right)$, where $h$ is any integer and $v^{\prime}$ is an integer which satisfies $v^{\prime} \equiv \pm 4^{ \pm 1} \bmod 23$.

Theorem 6.3 Suppose $N(U+0)=N(10)$ and $t \neq \pm 1$.

(1) If $N\left(U+\frac{t}{w}\right)=11 a 247\left(\frac{z}{v}= \pm \frac{19}{2}\right)$, then $\frac{t}{w}=\frac{19-10 v^{\prime}}{v^{\prime}-\left(19-10 v^{\prime}\right) h}$ and $U=\left(\frac{10}{10 h+1}\right)$, where $h$ is any integer and $v^{\prime}$ is an integer which satisfies $v^{\prime} \equiv \pm 2^{ \pm 1} \bmod 19$.

(2) If $N\left(U+\frac{t}{w}\right)=11 a 343\left(\frac{z}{v}= \pm \frac{31}{4}\right)$, then $\frac{t}{w}=\frac{31-10 v^{\prime}}{v^{\prime}-\left(31-10 v^{\prime}\right) h}$ and $U=\left(\frac{10}{10 h+1}\right)$, where $h$ is any integer and $v^{\prime}$ is an integer which satisfies $v^{\prime} \equiv \pm 4^{ \pm 1} \bmod 31$.

(3) If $N\left(U+\frac{t}{w}\right)=11 a 363\left(\frac{z}{v}= \pm \frac{35}{6}\right)$, then $\frac{t}{w}=\frac{35-10 v^{\prime}}{v^{\prime}-\left(35-10 v^{\prime}\right) h}$ and $U=\left(\frac{10}{10 h+1}\right)$, where $h$ is any integer and $v^{\prime}$ is an integer which satisfies $v^{\prime} \equiv \pm 6^{ \pm 1} \bmod 35$.

A tangle is a generalized $M$-tangle if it is ambient isotopic to a sum of rational tangles, or equivalently, it is obtained from a finite sum of rational tangles by a circle product with a finite sequence of integers. The above results can also be obtained by using the software TopoIce-R [8] within Knotplot [26]. This software implements the following theorems.

Theorem 6.4 (Ernst [11]) If $N(N+0)=N\left(\frac{a}{b}\right)$ and $N\left(U+\frac{t}{w}\right)=N\left(\frac{z}{v}\right)$ and if $|t|>1$, then $U$ is a generalized $M$-tangle. 
Theorem 6.5 (Darcy [7, Theorem 3]) $N(U+0)=N\left(\frac{a}{b}\right)$ and $N\left(U+\frac{t}{w}\right)=N\left(\frac{z}{v}\right)$ where $U$ is a generalized $M$-tangle if and only if the following hold.

(a) If $w \not \equiv \pm 1 \bmod t$, then there exists an integer, $b^{\prime}$ such that $b^{\prime} b^{ \pm 1}=1 \bmod a$, and for any integers $x$ and $y$ such that $b^{\prime} x-a y=1$,

$$
N\left(\frac{z}{v}\right)=N\left(\frac{t b^{\prime}+w a}{t y+w x}\right) .
$$

In this case, $U=\frac{a}{b^{\prime}}$ for all $b^{\prime}$ satisfying the above.

(b) If $w \equiv \varepsilon= \pm 1 \bmod t(w=h t+\varepsilon)$, then there exists relatively prime integers, $p$ and $q$, where $p$ may be chosen to be positive, such that,

$$
N\left(\frac{z}{v}\right)=N\left(\frac{t p(p b-q a)+\varepsilon a}{t q(p b-q a)+\varepsilon b}\right) .
$$

In this case, the solutions for $U$ are $\left(\frac{d a-j p}{p b-q a}+\frac{j}{p}\right) \circ(h, 0)$ and $\left(\frac{j}{p}+\frac{d a-j p}{p b-q a}\right) \circ(h, 0)$, for all $p, q$ satisfying the above, $d$ and $j$ are integers such that $p d-q j=1$ (note, the choice of $j$ and $d$ such that $p d-q j=1$ has no effect on $U$ ).

Corollary 6.6 (Darcy [7, Corollary 2]) Suppose $b x-a y=1, N\left(U+\frac{0}{1}\right)=N\left(\frac{a}{b}\right)$ and $N\left(U+\frac{t}{w}\right)=N\left(\frac{z}{v}\right)$ where $N\left(\frac{a}{b}\right)$ and $N\left(\frac{z}{v}\right)$ are unoriented 2-bridge knots or links. If $w \neq \equiv \pm 1$ or if $U$ is rational, then $\frac{t}{w}=\frac{x z-a v^{\prime}}{b v^{\prime}-y z-h t}$ and $U=\frac{a}{b+h a}$, or $\frac{t}{w}=\frac{b z-a v^{\prime}}{x v^{\prime}-y z-h t}$ and $U=\frac{a}{x+h a}$, where $v^{\prime}$ is any integer such that $v^{\prime} v^{ \pm 1}=1 \bmod z$. If $w \equiv \pm 1 \bmod t$, then $t$ divides $z \mp a$.

If $w \not \equiv \pm 1$ or if $U$ is rational and if $N\left(\frac{a}{b}\right)=N(2 k)$, then $\frac{t}{w}=\frac{z-2 k v^{\prime}}{v^{\prime}-\left(z-2 k v^{\prime}\right) h}$ and $U=\frac{2 k}{1+2 k h}$. If $w \equiv \varepsilon= \pm 1 \bmod t$, then $t, p$, and $p b-q a$ in part (b) of Theorem 6.5 are all factors of $z \mp a$. Note that if $U$ is not rational in Theorem 6.5, then both $|p|>1$ and $|p b-q a|>1$. We will use this to show that the conclusion (b) of Theorem 6.5 does not occur under the assumptions of Theorem $6.1,6.2$ or 6.3 .

Proof of Theorem 6.1 Suppose $k=3$ and $\frac{z}{v}=\frac{4 m n-1}{2 m}= \pm \frac{11}{2}$ or $\pm \frac{15}{4}$. If $U$ is rational, then Theorem 6.1 holds by Corollary 6.6. Suppose $U$ is not rational. Then $|t p(p-6 q)|=|z-6 \varepsilon|=5,9,17$ or 21 . But this is not possible since $|t|,|p|$, and $|p-6 q|$ are all greater than 1 .

Proof of Theorem 6.2 Suppose $k=4$ and $\frac{z}{v}=\frac{4 m n-1}{2 m}= \pm \frac{15}{2}$ or $\pm \frac{23}{4}$. If $U$ is rational, then Theorem 6.2 holds by Corollary 6.6. Suppose $U$ is not rational. Then $|t p(p-8 q)|=|z-8 \varepsilon|=7,15,23$ or 31 . But this is not possible since $|t|,|p|$, and $|p-8 q|$ are all greater than 1 . 
Proof of Theorem 6.3 Suppose $k=5$ and $\frac{z}{v}=\frac{4 m n-1}{2 m}= \pm \frac{19}{2}, \pm \frac{31}{4}$ or $\pm \frac{35}{6}$. If $U$ is rational, then Theorem 6.3 holds by Corollary 6.6. Suppose $U$ is not rational. Then $|t p(p-10 q)|=|z-10 \varepsilon|=9,21,25,29,41$ or 45 . Since $|t|,|p|$, and $|p-10 q|$ are all greater than 1 , the only possibility is that $|z-10 \varepsilon|=45$ and $|p(p-10 q)|=9$ or 15. But there is no integer solution to $|p(p-10 q)|=9$ or 15 where $|p|$ and $|p-10 q|$ are both greater than one.

The action of Xer recombination is believed to correspond to a $\left(-\frac{1}{3},-\frac{4}{3}\right)$ move. By Theorem 2.2, a $\left(-\frac{1}{3},-\frac{4}{3}\right)$ move is equivalent to a $\left(0, \frac{9}{9 l+5}\right)$ move for any integer $l$. Moreover, $N(U+0)=K_{1}$ and $N\left(U+\frac{9}{5}\right)=K_{2}$ if and only if $N([U \circ(1,2,0)]+$ $\left.\left(-\frac{1}{3}\right)\right)=K_{1}$ and $N\left([U \circ(1,2,0)]+\left(-\frac{4}{3}\right)\right)=K_{2}$, since $N(U+0)=N([U \circ(1,2,0) \circ$ $(-2,-1)]+0)=N([U \circ(1,2,0)]+[(0) \circ(-1,-2,0)])=N\left([U \circ(1,2,0)]+\left(-\frac{1}{3}\right)\right)$ and $N\left(U+\frac{9}{5}\right)=N\left(U+\left(-\frac{4}{3}\right) \circ(2,1)\right)=N\left([U \circ(1,2,0)]+\left(-\frac{4}{3}\right)\right)$. Then we obtain following corollaries from Theorem 6.1, 6.2 and 6.3.

Corollary 6.7 Suppose $N\left(U+\left(-\frac{1}{3}\right)\right)=N(6)$.

(1) If $N\left(U+\left(-\frac{4}{3}\right)\right)=7_{2}\left(\frac{z}{v}= \pm \frac{11}{2}\right)$, then it has no solution.

(2) If $N\left(U+\left(-\frac{4}{3}\right)\right)=7_{4}\left(\frac{z}{v}= \pm \frac{15}{4}\right)$, then $U=\left(-\frac{1}{3}\right)$.

Corollary 6.8 Suppose $N\left(U+\left(-\frac{1}{3}\right)\right)=N(8)$.

(1) If $N\left(U+\left(-\frac{4}{3}\right)\right)=9_{2}\left(\frac{z}{v}= \pm \frac{15}{2}\right)$, it has no solution.

(2) If $N\left(U+\left(-\frac{4}{3}\right)\right)=9_{5}\left(\frac{z}{v}= \pm \frac{23}{4}\right)$, then $U=\left(-\frac{1}{5}\right)$.

Corollary 6.9 Suppose $N\left(U+\left(-\frac{1}{3}\right)\right)=N(10)$.

(1) If $N\left(U+\left(-\frac{4}{3}\right)\right)=11 a 247\left(\frac{z}{v}= \pm \frac{19}{2}\right)$, then it has no solution.

(2) If $N\left(U+\left(-\frac{4}{3}\right)\right)=11 a 343\left(\frac{z}{v}= \pm \frac{31}{4}\right)$, then $U=\left(-\frac{1}{7}\right)$.

(3) If $N\left(U+\left(-\frac{4}{3}\right)\right)=11 a 363\left(\frac{z}{v}= \pm \frac{35}{6}\right)$, then it has no solution.

\section{Summary or conclusion or software}

Per theorems of Ernst [11] and Darcy [7], the system of tangle equations $N(U+$ $B)=N\left(\frac{a}{b}\right), N(U+E)=N\left(\frac{z}{v}\right)$ is easily solved when the $(B, E)$ move is equivalent to a $\left(0, \frac{t}{w}\right)$ move where $|t|>1$. The case when $|t|=1$ is much more difficult. A few special subcases when $|t|=1$ can be handled using results of Hirasawa and Shimokawa [17] and Kronheimer, Mrowka, Ozsváth and Szabó [21]. Our 
Theorem 3.1 handles the subcase when the move corresponds to a coherent banding and $N\left(\frac{a}{b}\right), N\left(\frac{z}{v}\right) \in\left\{N(2 k), N\left(\frac{4 m n-1}{2 m}\right)\right\}$. This subcase is particularly biologically relevant since $N\left(\frac{4 m n-1}{2 m}\right)$ includes the family of twist knots, and we also applied it to analyze the experimental results of Xer recombination acting on $(2,2 k)$-torus links (see Bath, Sherratt and Colloms [1]).

The software TopoIce-R [8] within Knotplot [26] solves the system of tangle equations $N(U+B)=N\left(\frac{a}{b}\right), N(U+E)=N\left(\frac{z}{v}\right)$ when $U$ is a generalized $M$-tangle (that is, ambient isotopic to a sum of rational tangles) and $B$ and $E$ are rational tangles. When the $(B, E)$ move is equivalent to a $\left(0, \frac{t}{w}\right)$ move where $|t|>1$, then $U$ must be a generalized $M$-tangle by Theorem 6.4. However, other types of tangles can be solutions for $U$ when $|t|=1$ (see Darcy [6]). These solutions are not currently found by TopoICE-R. Note that the solutions for $U$ in Theorem 3.1 in which $|t|=1$ are generalized $M$-tangles. Hence for the cases in Theorem 3.1, TopoIce-R finds all solutions to this system of tangle equations. The solutions found by TopoICE-R correspond to performing surgery on a $(p, q)$ torus knot in the double branch cover of $N\left(\frac{a}{b}\right)$.

\section{Acknowledgments}

Kai Ishihara is partially supported by EPSRC Grant EP/H031367/1 to Dorothy Buck. Koya Shimokawa is partially supported by KAKENHI 18540069 and 22540066. This research was supported in part by a grant from the Joint DMS/NIGMS Initiative to Support Research in the Area of Mathematical Biology (NSF 0800285) to Isabel Darcy. The authors gratefully acknowledge the support and hospitality provided by of the Institute for Mathematics and its Applications (IMA) as well as the Centro di Ricerca Matematica Ennio De Giorgi.

\section{References}

[1] J Bath, D Sherratt, S Colloms, Topology of Xer recombination on catenanes produced by lambda integrase, J. Mol. Biol. 289 (1999) 873-883

[2] D Buck, C Verjovsky Marcotte, Tangle solutions for a family of DNA-rearranging proteins, Math. Proc. Cambridge Philos. Soc. 139 (2005) 59-80 MR2155505

[3] S D Colloms, J Bath, D J Sherratt, Topological selectivity in Xer site-specific recombination, Cell 88 (1997) 855-864

[4] J H Conway, An enumeration of knots and links, and some of their algebraic properties, from: "Computational Problems in Abstract Algebra (Proc. Conf., Oxford, 1967)", Pergamon, Oxford (1970) 329-358 MR0258014 
[5] N J Crisona, R L Weinberg, B J Peter, D W Sumners, N Cozzarelli, The topological mechanism of phage $\lambda$ integrase, J. Mol. Biol. 289 (1999) 747-775

[6] I K Darcy, Biological distances on DNA knots and links: applications to XER recombination, J. Knot Theory Ramifications 10 (2001) 269-294 MR1822492 Knots in Hellas '98, Vol. 2 (Delphi)

[7] I K Darcy, Solving unoriented tangle equations involving 4-plats, J. Knot Theory Ramifications 14 (2005) 993-1005 MR2196644

[8] I K Darcy, R G Scharein, TopoICE-R: 3D visualization modeling the topology of DNA recombination, Bioinformatics 22 (2006) 1790-1791

[9] I K Darcy, D W Sumners, Rational tangle distances on knots and links, Math. Proc. Cambridge Philos. Soc. 128 (2000) 497-510 MR1744106

[10] A Deruelle, K Miyazaki, K Motegi, Networking Seifert surgeries on knots III, preprint (2011) Available at http://www.math.chs.nihon-u.ac.jp/ motegi/ DMMnetwork3.pdf

[11] C Ernst, Tangle equations, J. Knot Theory Ramifications 5 (1996) 145-159 MR1395775

[12] C Ernst, D W Sumners, A calculus for rational tangles: applications to DNA recombination, Math. Proc. Cambridge Philos. Soc. 108 (1990) 489-515 MR1068451

[13] C Ernst, D W Sumners, Solving tangle equations arising in a DNA recombination model, Math. Proc. Cambridge Philos. Soc. 126 (1999) 23-36 MR1681651

[14] J R Goldman, L H Kauffman, Rational tangles, Adv. in Appl. Math. 18 (1997) 300332 MR1436484

[15] I Grainge, M Bregu, M Vazquez, V Sivanathan, S Ip, D Sherratt, Unlinking chromosomes catenated in vivo by site-specific recombination, EMBO J. 26 (2007) 4228-4238

[16] A Hatcher, W Thurston, Incompressible surfaces in 2-bridge knot complements, Invent. Math. 79 (1985) 225-246 MR778125

[17] M Hirasawa, K Shimokawa, Dehn surgeries on strongly invertible knots which yield lens spaces, Proc. Amer. Math. Soc. 128 (2000) 3445-3451 MR1676336

[18] T C Hodgman, H Griffiths, D K Summers, Nucleoprotein architecture and ColE1 dimer resolution: a hypothesis, Mol. Microbiol. 29 (1998) 545-558

[19] S C Y Ip, M Bregu, F-X Barre, D J Sherratt, Decatenation of DNA circles by FtsKdependent Xer site-specific recombination, EMBO J. 22 (2003) 6399-6407

[20] T Kobayashi, Uniqueness of minimal genus Seifert surfaces for links, Topology Appl. 33 (1989) 265-279 MR1026928

[21] P Kronheimer, T Mrowka, P Ozsváth, Z Szabó, Monopoles and lens space surgeries, Ann. of Math. 165 (2007) 457-546 MR2299739 
[22] H R Morton, R J Hadji, Conjugacy for positive permutation braids, Fund. Math. 188 (2005) 155-166 MR2191944

[23] K Motegi, Knot types of satellite knots and twisted knots, from: "Lectures at KNOTS '96 (Tokyo)", Ser. Knots Everything 15, World Sci. Publ., River Edge, NJ (1997) 73-93 MR1474519

[24] K Murasugi, On a certain numerical invariant of link types, Trans. Amer. Math. Soc. 117 (1965) 387-422

[25] K Murasugi, Knot theory and its applications, Birkhäuser, Boston, MA (1996) MR1391727 Translated from the 1993 Japanese original by Bohdan Kurpita

[26] R G Scharein, Interactive topological drawing, $\mathrm{PhD}$ thesis, University of British Columbia (1998) MR2697507

[27] M Scharlemann, Smooth spheres in $\mathbb{R}^{4}$ with four critical points are standard, Invent. Math. 79 (1985) 125-141 MR774532

[28] M Scharlemann, A Thompson, Link genus and the Conway moves, Comment. Math. Helv. 64 (1989) 527-535 MR1022995

[29] K Shimokawa, K Ishihara, M Vazquez, Tangle analysis of DNA unlinking by the Xer/FtsK system, Bussei Kenkyu 92 (2009) 89-92

[30] D W Sumners, C Ernst, S J Spengler, N Cozzarelli, Analysis of the mechanism of DNA recombination using tangles, Q. Rev. Biophys. 28 (1995) 253-313

[31] A Thompson, Knots with unknotting number one are determined by their complements, Topology 28 (1989) 225-230 MR1003584

[32] M Vazquez, D W Sumners, Tangle analysis of Gin site-specific recombination, Math. Proc. Cambridge Philos. Soc. 136 (2004) 565-582 MR2055047

Department of Mathematics and Applied Mathematical and Computational Sciences University of Iowa, 14 MLH, Iowa City IA 52242, USA

Department of Mathematics, Imperial College London

London SW7 2AZ, UK

Pyxis Solutions

New York NY 10004, USA

Department of Mathematics, Saitama University

Saitama 338-8570, Japan

idarcy.math@gmail.com, k.ishihara@imperial.ac.uk, medukishore@gmail.com, kshimoka@rimath.saitama-u.ac.jp

http://www.math.uiowa.edu/ idarcy/,

http://www2.imperial.ac.uk/ kishihar/, http://www.rimath.saitama-u.ac.jp/lab.en/kshimoka/index-e.html

Received: 29 September $2011 \quad$ Revised: 2 February 2012 